Publ. Mat. (2002), 39-56

Proceedings of the $6^{\text {th }}$ International Conference on Harmonic Analysis

and Partial Differential Equations. El Escorial, 2000.

\title{
DIRECTIONAL OPERATORS AND MIXED NORMS
}

\author{
JAVIER DUOANDIKOETXEA
}

Dedicated to the memory of Chicho Guadalupe, a friend of many of us

Abstract

We present a survey of mixed norm inequalities for several directional operators, namely, directional Hardy-Littlewood maximal functions and Hilbert transforms (both appearing in the method of rotations of Calderón and Zygmund), $X$-ray transforms, and directional fractional operators related to Riesz type potentials with variable kernel. In dimensions higher than two several interesting questions remain unanswered.

\section{Introduction}

\subsection{The method of rotations for singular integrals.}

Directional operators in $\mathbf{R}^{n}$ are defined as the action of one-dimensional operators on the restriction of functions to lines. The model operators are the directional Hardy-Littlewood maximal function

$$
M f(x, u)=\sup _{h>0} \frac{1}{h} \int_{0}^{h}|f(x-t u)| d t
$$

and the directional Hilbert transform

$$
H f(x, u)=\mathrm{p} . \mathrm{v} . \int_{-\infty}^{\infty} \frac{f(x-t u)}{t} d t,
$$

defined for $x \in \mathbf{R}^{n}$ and $u \in S^{n-1}$. Fubini's theorem implies that

$$
\|M f(\cdot, u)\|_{p},\|H f(\cdot, u)\|_{p} \leq C(p)\|f\|_{p}
$$

where $C(p)$ is a bound for the $L^{p}$ norm of the one-dimensional HardyLittlewood maximal operator and the Hilbert transform.

2000 Mathematics Subject Classification. 42B20, 44A12.

Key words. Directional Hardy-Littlewood maximal functions, directional Hilbert transform, $X$-ray transform, mixed norms, potentials.

Partially supported by grant EB051/99 of the Universidad del País Vasco/Euskal Herriko Unibertsitatea. 
Both operators together with the directional maximal Hilbert transform (whose definition will be easily guessed by the reader) appear in the method of rotations introduced by Calderón and Zygmund [CZ1] to study singular integral operators with variable kernel, namely,

$$
T_{\Omega} f(x)=\text { p.v. } \int_{\mathbb{R}^{n}} \frac{\Omega\left(x, y^{\prime}\right)}{|y|^{n}} f(x-y) d y
$$

where $y^{\prime}=y /|y|$. Suppose that $\Omega$ is odd in its second variable, then the singular integral can be represented as

$$
T_{\Omega} f(x)=\frac{1}{2} \int_{S^{n-1}} \Omega(x, u) H f(x, u) d \sigma(u) .
$$

Consider first the case when $\Omega$ is independent of $x$ which is quite well-known. From the representation (1.2) it is clear that

$$
\left\|T_{\Omega} f\right\|_{p} \leq \frac{1}{2} C(p)\|\Omega\|_{1}\|f\|_{p}
$$

where $\|\Omega\|_{1}$ is the norm on the unit sphere. Since (1.2) is valid only for odd $\Omega$, the case when $\Omega$ is even needs a different approach which already appeared in $[\mathbf{C Z 1}]$; the conclusion is that the $L^{p}$-boundedness of $T_{\Omega}$ holds when $\Omega$ has mean value zero (which is a necessary condition) and the condition $\Omega \in L^{1}$ is replaced by $\Omega \in L \log L$ (in particular, $\Omega \in L^{r}$ for some $r>1$ suffices).

If $\Omega$ depends on $x$, the situation is more complicated. Define

$$
N_{r}=\left(\int_{S^{n-1}}|\Omega(x, u)|^{r^{\prime}} d \sigma(u)\right)^{1 / r^{\prime}}
$$

and apply Hölder's inequality to majorize (1.2) by

$$
\left|T_{\Omega} f(x)\right| \leq \frac{1}{2} N_{r}\left(\int_{S^{n-1}}|H f(x, u)|^{r} d \sigma(u)\right)^{1 / r} .
$$

Then, if $N_{r}$ is finite, $T_{\Omega}$ is bounded on $L^{p}$ for odd $\Omega$ if the following inequality holds:

$$
\left(\int_{\mathbf{R}^{n}}\left(\int_{S^{n-1}}|H f(x, u)|^{r} d \sigma(u)\right)^{p / r} d x\right)^{1 / p} \leq C_{p, r}\|f\|_{p} .
$$

As a consequence of (1.1), if $1<r<\infty$, (1.4) holds for $p \geq r$. We are interested in values $p<r$ for which (1.4) holds. 
For a function $F$ defined on $\mathbf{R}^{n} \times S^{n-1}$ and $1 \leq p, r \leq \infty$ we define the mixed norm of $F$ in $L^{p}\left(L^{r}\right)$ as

$$
\|F\|_{L^{p}\left(L^{r}\right)}=\left(\int_{\mathbf{R}^{n}}\|F(x, \cdot)\|_{L^{r}\left(S^{n-1}\right)}^{p} d x\right)^{1 / p},
$$

with the usual modification when $p=\infty$. With this notation Inequality (1.4) and its counterpart for the maximal function are written as

$$
\|H f\|_{L^{p}\left(L^{r}\right)},\|M f\|_{L^{p}\left(L^{r}\right)} \leq C_{p, r}\|f\|_{p} .
$$

The study of these inequalities will be considered in Section 2 .

\subsection{The $X$-ray transform.}

Given a smooth function $f$ in $\mathbf{R}^{n}$ we define its $X$-ray transform as

$$
X f(x, u)=\int_{-\infty}^{\infty} f(x-t u) d t
$$

for $x \in \mathbf{R}^{n}$ and $u \in S^{n-1}$. Although we define the operator on $\mathbf{R}^{n} \times$ $S^{n-1}$, it is constant on lines, so that actually we can consider only those $x$ which are orthogonal to $u$. The interesting mixed norm inequalities in this setting are

$$
\left(\int_{S^{n-1}}\left(\int_{u^{\perp}}|X f(x, u)|^{q} d \lambda_{u^{\perp}}(x)\right)^{r / q} d \sigma(u)\right)^{1 / r} \leq C_{p, q, r}\|f\|_{p}
$$

where $d \lambda_{u^{\perp}}$ is the $(n-1)$-dimensional Lebesgue measure on $u^{\perp}$. Section 3 is devoted to the study of this kind of mixed norm inequalities which apart from their own interest will be useful for the other operators studied in this paper.

\subsection{The method of rotations for potential type operators.}

Given $\Omega$ as before, we can define operators associated to $\Omega$ with the homogeneity of the Riesz potentials, that is,

$$
I_{\alpha, \Omega} f(x)=\int_{\mathbf{R}^{n}} \frac{\Omega\left(x, y^{\prime}\right)}{|y|^{n-\alpha}} f(x-y) d y, \quad 0<\alpha<n .
$$

Using polar coordinates we have a representation similar to the righthand side of (1.2),

$$
I_{\alpha, \Omega} f(x)=\int_{S^{n-1}} \Omega(x, u) \int_{0}^{\infty} f(x-t u) t^{\alpha-1} d t d \sigma(u) .
$$


We define directional Riesz potentials for $0<\alpha<n$ as

$$
I_{\alpha} f(x, u)=\int_{-\infty}^{\infty} f(x-t u)|t|^{\alpha-1} d t .
$$

For $\alpha=1$ this operator coincides with the $X$-ray transform. When $N_{r}$ (defined as in (1.3)) is finite, the boundedness properties of $I_{\alpha, \Omega}$ from $L^{p}$ to $L^{q}$ are deduced from the following mixed norm inequalities

$$
\left\|I_{\alpha} f\right\|_{L^{q}\left(L^{r}\right)} \leq C_{p, q, r}\|f\|_{p}
$$

which we will study in Section 4. Given $p$ there is just one value of $q$ for which (1.10) is possible, so that actually there are only two free indexes in this inequality.

\subsection{Some variants.}

Appropriate counterexamples show that there are values of $p, q$ and $r$ for which (1.5), (1.6) and (1.10) are not possible. In general, only in the two dimensional case full answers are known, and the problems remain open for $n \geq 3$ in part of the expected range of boundedness. In Section 5 we study all the operators when they act on radial functions; although the range of values of $p, q$ and $r$ for which boundedness holds is larger than for general functions, we are able to prove sharp results.

Finally, in Section 6 we review some results on maximal operators associated to an arbitrary set of directions. Several situations are possible depending on the set of parameters and again many questions remain open.

\section{The maximal operator and the Hilbert transform}

\subsection{The conjecture and the theorem.}

Taking as $f$ the characteristic function of a ball, the size of $M f(x, u)$ and $H f(x, u)$ for big $x$ is of the order of $|x|^{-1}$ when $u$ is in a ball of $S^{n-1}$ of radius $|x|^{-1} / 2$; then the left-hand side of (1.5) is finite only if

$$
\frac{n}{p}-\frac{n-1}{r}<1
$$

On the other hand, the characteristic function of a Besicovitch type set excludes the case $r=\infty$ (see $[\mathbf{S 2}$, Chapter $\mathrm{X}]$ ). We formulate the following conjecture.

Conjecture 2.1. Inequality (1.5) holds for $M$ and $H$ if and only if

$$
\frac{n}{p}-\frac{n-1}{r}<1 \quad \text { and } \quad 1 \leq r<\infty \text {. }
$$




\section{Theorem 2.2.}

(i) The conjecture holds true for $n=2$.

(ii) The conjecture holds true for $n \geq 3$ if $1<p \leq(n+1) / 2$.

By interpolation the boundedness of $M$ and $H$ for $(n+1) / 2<p<\infty$ and $r<2 p$ is deduced from (ii).

As mentioned in the introduction the result for $r \leq p$ is immediate, so that we only need to consider the case $r>p$.

\subsection{The $L^{2}$ estimate.}

The first nontrivial result was proved by Calderón and Zygmund in $[\mathbf{C Z 2}]$. They showed that the conjecture holds true for the Hilbert transform when $n=2$ and also for $n \geq 3$ if $1<p \leq 2$. Actually they proved the best possible results for $p=2$ in all dimensions and RieszThorin interpolation provides the rest. Their proof was based on the use of spherical harmonics. An alternative proof is due to M. Christ and consists in proving that $H$ is bounded from $L^{2}$ to $L^{2}\left(L_{\beta}^{2}\right)$ for $\beta<1 / 2$ (here $L_{\beta}^{2}$ is the Sobolev space defined on $S^{n-1}$, see [S1, Chapter IV]). Since we are in a Hilbert space setting, the norm in $L^{2}\left(L_{\beta}^{2}\right)$ of $H f$ coincides with the norm of $\widehat{H f}$ where the Fourier transform is taken in the $x$ variable. But

$$
\widehat{H f}(\xi, u)=-\pi i \operatorname{sgn}(\xi \cdot u) \hat{f}(\xi)
$$

and $\operatorname{sgn}(\xi \cdot u)$ as a function of $u$ is in $L_{\beta}^{2}\left(S^{n-1}\right)$ if and only if $\beta<1 / 2$ (the norm is clearly independent of $\xi$ ). Using the Sobolev embedding theorem, $L_{\beta}^{2}$ is continuously included in $L^{r}$ for $1 / r=1 / 2-\beta /(n-1)$ and this proves the result.

For the maximal operator the first nontrivial result is due to R. Fefferman $[\mathbf{F 2}]$ who proved the best results for $r=2$ in all dimensions (that is, for $1<p \leq 2 n /(n+1))$. Interpolation gives some non optimal results for $p>2 n /(n+1)$. His result was improved by M. Cowling and G. Mauceri $[\mathbf{C M}]$ who extended it to the same range given by Calderón and Zygmund for the Hilbert transform, that is, the optimal result was proved to hold for $1<p \leq 2$ in all dimensions. By interpolation with the trivial estimate in $L^{\infty}$ this result implies the full conjecture if $n=2$ and some partial results for $n \geq 3$ and $p>2$. Next we sketch the alternative proof given in [CDR].

To work with the maximal operator we consider smooth functions $\varphi$ (defined on $\mathbf{R}$ ) and $\phi$ (defined on $\mathbf{R}^{n}$ and radial) of compact support, nonnegative, with integral one, and such that $\varphi(0)>0$. Define $\varphi_{k}(t)=$ 
$2^{-k} \varphi\left(2^{-k} t\right)$ and $\phi_{k}(x)=2^{-k n} \phi\left(2^{-k} x\right)$, and introduce

$$
T_{k} f(x, u)=\int_{-\infty}^{\infty} f(x-t u) \varphi_{k}(t) d t
$$

and

$$
A_{k} f(x, u)=\int_{-\infty}^{\infty} f(x-t u) \varphi_{k}(t) d t-\int_{\mathbf{R}^{n}} f(x-y) \phi_{k}(y) d y .
$$

If $f$ is nonnegative

$$
M f(x, u) \leq C \sup _{k \in \mathbf{Z}} T_{k} f(x, u) \leq C\left(\sup _{k \in \mathbf{Z}}\left|A_{k} f(x, u)\right|+f^{*}(x)\right)
$$

where $f^{*}$ denotes the ( $n$-dimensional) Hardy-Littlewood maximal function of $f$.

As in the Hilbert transform case we work with Sobolev spaces in $u$. The estimate

$$
\left\|A_{k} f\right\|_{L^{2}\left(L_{\beta}^{2}\right)}^{2} \leq C \int_{\mathbf{R}^{n}}|\hat{f}(\xi)|^{2} \max \left(2^{k}|\xi|, 2^{-k}|\xi|\right)^{-\alpha} d \xi
$$

with $\alpha=1-2 \beta$ (see [CDR]) gives the $L^{2}$-boundedness for $\beta<1 / 2$ and using the Sobolev embedding as before we recover the result obtained by Cowling and Mauceri.

\section{3. $L^{p}$ estimates, $p>2$.}

Results for $p>2$ were improved in the paper by M. Christ, J. Duoandikoetxea and J. L. Rubio de Francia [CDR]. They are contained in the statement of Theorem 2.2 and have not been improved since then.

An interesting fact proved in $[\mathbf{C D R}]$ is that the result for the maximal operator provides the result for the Hilbert transform. To be precise the following lemma holds.

Lemma 2.3. If $M$ satisfies (1.5) for some $\left(p_{0}, r_{0}\right)$ and $R$ is any onedimensional operator which is bounded on $L^{p}(w, \mathbf{R})$ for all $w \in A_{p}(\mathbf{R})$, then the directional operator defined through $R$ satisfies a mixed norm inequality like (1.5) in $L^{p_{0}}\left(L^{r}\right)$ with $r<r_{0}$.

$A_{p}$ refers to the Muckenhoupt class of weights for which the HardyLittlewood maximal operator is bounded on the weighted space $L^{p}(w)$. The proof of this lemma is similar to the proof of part of the extrapolation theorem as presented for instance in $[\mathbf{D}]$. Since the Hilbert transform and the maximal Hilbert transform satisfy the required weighted inequalities, proving Theorem 2.2 for the maximal operator gives the result for the Hilbert transform and the maximal Hilbert transform. 
To improve the results for $p_{0}>2$ it is enough to show that for some $C$ independent of $k$ and some $r_{0}$ not covered in the previous result, the following mixed norm inequality holds

$$
\left\|T_{k} f\right\|_{L^{p_{0}\left(L^{r_{0}}\right)}} \leq C\|f\|_{p_{0}} .
$$

To see this define a usual Littlewood-Paley family of operators $\left\{S_{j}\right\}$ as $\left(S_{j} f\right)^{\wedge}(\xi)=\psi\left(2^{j}|\xi|\right) \hat{f}(\xi)$ where $\psi$ is a radial $\mathcal{C}^{\infty}$ function supported in $\{1 / 2<|\xi|<2\}$ and such that $\sum_{j} \psi\left(2^{j}|\xi|\right)=1$ for $\xi \neq 0$; let

$$
B_{j} f=\sup _{k}\left|A_{k}\left(S_{j+k} f\right)\right|,
$$

so that

$$
\sup _{k}\left|A_{k} f(x, u)\right| \leq \sum_{j} B_{j} f(x, u) .
$$

Using (2.3) we get

$$
\left\|B_{j} f\right\|_{L^{2}\left(L^{r}\right)} \leq C 2^{-\alpha|j|}\|f\|_{2} \quad \text { for } r<\frac{2 n-2}{n-2},
$$

and from (2.4),

$$
\left\|B_{j} f\right\|_{L^{p_{0}\left(L^{r_{0}}\right)}} \leq C\|f\|_{p_{0}}
$$

is deduced. Interpolating and using (2.5) and (2.2) we deduce a boundedness result for $M$.

Theorem 2.2 is obtained proving (2.4) for $p_{0}=(n+1) / 2$ and $r_{0}=n+1$. By a dilation argument we can reduce ourselves to $k=0$; for nonnegative $f, T_{0} f$ is bounded by the $X$-ray transform and the required inequality is obtained using Theorem 3.2 below. The details can be seen in [CDR].

\subsection{Application to the Kakeya maximal operator.}

Directional maximal operators can be used to control averages over starlike sets. More precisely, let $E$ be a set starlike with respect to the origin, described as $E=\left\{r u: 0 \leq r \leq R(u), u \in S^{n-1}\right\}$, then

$$
\begin{aligned}
\frac{1}{|E|} \int_{E}|f(x-y)| d y & =\frac{1}{|E|} \int_{S^{n-1}} \int_{0}^{R(u)}|f(x-r u)| r^{n-1} d r d \sigma(u) \\
& \leq \frac{1}{|E|} \int_{S^{n-1}} R(u)^{n} M f(x, u) d \sigma(u) \\
& \leq \frac{\sup _{u} R(u)^{n / r}}{|E|^{1 / r}}\left(\int_{S^{n-1}} M f(x, u)^{r} d \sigma(u)\right)^{1 / r}
\end{aligned}
$$


Defining the eccentricity of a set $E$ as the infimum of the quotients $|B| /|E|$ for all balls $B$ containing $E$, the first term in the right-hand side can be replaced by the eccentricity of $E$ raised to the power $1 / r$. In particular, we get an expression which majorizes the maximal function over all the sets with a fixed eccentricity.

An interesting example is the Kakeya maximal operator $\mathcal{M}_{N}$ which takes the supremum over the averages on all paralellepipeds of sides $a \times$ $a \times \cdots \times a \times N a$ for a fixed $N$ and variable $a>0$. The conjecture is that this operator is bounded on $L^{p}$ with norm $C(N, p)$ majorized as

$$
C(N, p) \leq \begin{cases}C(p)(\log N)^{a(p)}, & \text { for some } a(p)>0 \text { if } p \geq n \\ C(p) N^{n / p-1}(\log N)^{a(p)}, & \text { for some } a(p) \geq 0 \text { if } 1<p<n\end{cases}
$$

Using the preceding pointwise bound and the fact that the eccentricity of the sets involved in the definition of Kakeya maximal function is $N^{n-1}$ we have

$$
\mathcal{M}_{N} f(x) \leq C N^{(n-1) / r}\left(\int_{S^{n-1}} M f(x, u)^{r} d \sigma(u)\right)^{1 / r} .
$$

Then to prove (2.6) for some $p<n$ (with $a(p)=0$ ) it would be enough to have $(1.5)$ for $(n-1) / r=n / p-1$. But this is exactly the endpoint condition in Conjecture 2.1 and it is false. Nevertheless, since we accept to loose a power of $\log N$, it suffices to have (1.5) for all $r$ with $(n-1) / r>n / p-1$ provided that $C_{p, r}$ grows as a negative power of $r^{-1}-r_{n}(p)^{-1}$, where $r_{n}(p)$ satisfies

$$
\frac{n}{p}-\frac{n-1}{r_{n}(p)}=1 \quad \text { if } p<n \quad \text { and } \quad r_{n}(p)=\infty \quad \text { if } p \geq n .
$$

Using Theorem 2.2 the full conjecture is deduced for $n=2$ (a result due to A. Córdoba $[\mathbf{C o 1}])$ and also for the range $1<p \leq(n+1) / 2$ when $n \geq 3$.

This relation between Theorem 2.2 and the boundedness of the Kakeya maximal function can be thought as a measure of its difficulty. Still, it is worth pointing out that (2.6) has been established for $p<(n+2) / 2$ by T. Wolff (see $[\mathbf{W 1}$ ) and also the survey [W2]) using a different method.

\section{The $X$-ray transform}

\subsection{The conjecture and the theorem.}

The $X$-ray transform has been defined in Subsection 1.2 and we look for inequalities of the type (1.6). Although apparently we have three 
parameters, $p, q$ and $r$, a dilation argument (replacing $f(x)$ by $f(\lambda x)$ ) gives the necessary condition

$$
\frac{n}{p}-\frac{n-1}{q}=1
$$

so that it is enough to determine the values of $p$ and $r$. This condition does not make sense if $p>n$, and actually $p=n$ is also excluded (take $f(x)=(1+|x|)^{-1}(\log |x|)^{-a}$ with $\left.1 / n<a<1\right)$. If we consider as $f$ the characteristic function of a paralellepiped of sides $1 \times \epsilon \times \cdots \times \epsilon$ and make $\epsilon$ tend to 0 , the condition

$$
r \leq(n-1) p^{\prime}
$$

appears. Altogether we formulate the following conjecture.

Conjecture 3.1. Inequality (1.6) holds if and only if

$$
1 \leq p<n, \quad \frac{n}{p}-\frac{n-1}{q}=1, \quad \text { and } \quad r \leq(n-1) p^{\prime} .
$$

Theorem 3.2. Conjecture 3.1 holds true if $n=2$ or if $n \geq 3$ and $1 \leq p \leq(n+1) / 2$.

Partial results were given by A. P. Calderón $[\mathbf{C}]$. The general result in two dimensions was proved by D. M. Oberlin and E. M. Stein [OS] using analytic interpolation; actually they proved an $n$-dimensional result for the Radon transform which is the analogue of the $X$-ray transform defined replacing lines by hyperplanes (so that $X$-ray and Radon transforms coincide for $n=2)$. In higher dimensions, S. W. Drury [Dr1] proved the result for $1<p<(n+1) / 2$ with strict inequality in (3.1) and M. Christ $[\mathbf{C h}]$ was able to prove the end-point result $p=(n+1) / 2$, $r=n+1$ which gives the theorem; the range $(n+1) / 2<p<n$ is open (see a related result for $n=3$ in [W3]). [Dr2] is an interesting survey for the more general $k$-plane transform $(k=1$ is the $X$-ray transform and $k=n-1$ is the Radon transform).

Notice first that the case $p=1, q=1, r=\infty$ is trivial due to Fubini's theorem and that it is enough to prove the estimates for the values of $p$ and $r$ on the line $r=(n-1) p^{\prime}$.

\subsection{The $L^{2}$ estimate.}

The two-dimensional result can be handled via Fourier transform and Sobolev imbedding as follows. Given $f$ in $\mathbf{R}^{2}$ its $X$-ray transform is

$$
X f(s, \theta)=\int_{-\infty}^{\infty} f(s \sin \theta+t \cos \theta,-s \cos \theta+t \sin \theta) d t
$$


for $s \in \mathbf{R}$ and $\theta \in[0,2 \pi)$. We claim that the $X$-ray transform is bounded from $L^{2}$ to $L^{2}\left(L_{1 / 2}^{2}\right)$ (since the mixed norm in (1.6) is taken in reverse order, the Sobolev space is in the $x$ variable). Considering the Fourier transform of $X f(s, \theta)$ with respect to $s$,

$$
(X f)^{\wedge}(\rho, \theta)=\int_{-\infty}^{\infty} X f(s, \theta) e^{-2 \pi i \rho s} d s,
$$

the mixed norm in $L^{2}\left(L_{1 / 2}^{2}\right)$ is given by

$$
\int_{0}^{2 \pi} \int_{-\infty}^{\infty}\left|(X f)^{\wedge}(\rho, \theta)\right|^{2}|\rho| d \rho d \theta .
$$

An easy calculation shows that

$$
(X f)^{\wedge}(\rho, \theta)=\hat{f}(\rho \sin \theta,-\rho \cos \theta),
$$

where $\hat{f}$ denotes the Fourier transform of $f$ in $\mathbf{R}^{2}$. The claim follows.

Interpolating with the trivial estimate $L^{1} \rightarrow L^{\infty}\left(L^{1}\right)$ we deduce the boundedness from $L^{p}$ to $L^{p^{\prime}}\left(L_{1 / p^{\prime}}^{p}\right)$ for $1<p<2$. The Sobolev imbedding gives $L_{1 / p^{\prime}}^{p} \subset L^{q}$ for $1 / q=2 / p-1$, ending the proof. (Actually one can perform a similar approach for $n \geq 3$ but the mixed norm inequality is far from being sharp.)

\subsection{The unmixed estimate.}

The best we know in higher dimensions is due to M. Christ who proved the sharp unmixed estimate (by unmixed we mean the case $q=r$, which is not properly a mixed norm). According to Conjecture 3.1 such an estimate can only hold for $1 \leq p \leq(n+1) / 2$. Christ's proof is based on a multilinear estimate and is presented next.

The key fact is that the multilinear form

$$
A\left(f_{0}, f_{1}, \ldots, f_{n}\right)=\int_{S^{n-1}} \int_{u^{\perp}} \prod_{j=0}^{n}\left(\int_{-\infty}^{\infty} f_{j}(x-t u) d t\right) d \lambda_{u^{\perp}}(x) d \sigma(u)
$$

is bounded from $L^{1} \times L^{n, 1} \times \cdots \times L^{n, 1}$ to $\mathbf{C}$. Here $L^{n, 1}$ is the Lorentz space whose definition and properties can be found in [BS]. It is enough to prove that

$$
\begin{array}{r}
\int_{S^{n-1}} \int_{u^{\perp}}\left(\int_{-\infty}^{\infty} f_{0}\left(x-t_{0} u\right) d t_{0}\right)\left(\int_{-\infty}^{\infty} f_{1}\left(x-t_{1} u\right) d t_{1}\right)^{n} d \lambda_{u^{\perp}}(x) d \sigma(u) \\
\leq C\left\|f_{0}\right\|_{1}\left\|f_{1}\right\|_{n, 1}^{n} .
\end{array}
$$


Write $y=x-t_{0} u$ gathering the second and third integral of the left-hand side to get

$$
\int_{S^{n-1}} \int_{\mathbf{R}^{n}} f_{0}(y)\left(\int_{-\infty}^{\infty} f_{1}(y-t u) d t\right)^{n} d y d \sigma(u)
$$

(after a translation in the inner integral). Then the result follows if we show that

$$
\int_{S^{n-1}}\left(\int_{-\infty}^{\infty} f(y-t u) d t\right)^{n} d \sigma(u) \leq C\|f\|_{n, 1}^{n} .
$$

It is enough to prove this result for characteristic functions (see $[\mathbf{B S}]$ ). Let $E$ be a measurable set in $\mathbf{R}^{n}$ of finite measure and $\chi_{E}$ its characteristic function; assuming without loss of generality that $y=0$ and using polar coordinates,

$$
\begin{aligned}
|E|=\int_{S^{n-1}}\left(\int_{0}^{\infty} \chi_{E}(t u) t^{n-1}\right. & d t) d \sigma(u) \\
& \geq \frac{1}{n} \int_{S^{n-1}}\left(\int_{0}^{\infty} \chi_{E}(t u) d t\right)^{n} d \sigma(u),
\end{aligned}
$$

where the inequality holds because

$$
|J|^{n} \leq n \int_{0}^{\infty} \chi_{J}(t) t^{n-1} d t
$$

for any set $J$ contained in $(0, \infty)$ (the right-hand side is minimum if $J=(0,|J|)$ due to the fact that $t^{n-1}$ is increasing $)$.

Moving the position of $L^{1}$ we have $n+1$ estimates, and applying multilinear interpolation to them we deduce that the above-mentioned multilinear form is bounded from $L^{p_{1}} \times L^{p_{2}} \times \cdots \times L^{p_{n+1}}$ to $\mathbf{C}$ when $1<p_{j}<n$ and $\sum_{j} 1 / p_{j}=2$. The case $p_{j}=(n+1) / 2$ for all $j$ gives the desired estimate and ends the proof of Theorem 3.2.

\section{Potential like operators}

\subsection{The conjecture and the theorem.}

The usual dilation argument (replace $f(x)$ with $f(\lambda x)$ ) and counterexamples (the characteristic functions of a ball and of $[-1,1] \times[-\delta, \delta]^{n-1}$ ) provide the necessary conditions to formulate our conjecture

Conjecture 4.1. Inequality (1.10) holds true if and only if $1<p<n / \alpha$ and

$$
\frac{n}{p}-\frac{n}{q}=\alpha, \quad \frac{n}{p}-\frac{n-1}{r}<1 \quad \text { and } \quad r \geq \frac{n}{\alpha} .
$$

The following theorem was proved in $[\mathbf{D O}]$. 
Theorem 4.2. The preceding conjecture holds true if $n=2$ or if $n \geq 3$ and $\alpha \geq n /(n+1)$. If $n \geq 3$ and $\alpha<n /(n+1)$ then Inequality (1.10) holds if moreover $r<2 p$.

\subsection{The key estimates.}

To prove the theorem we use three key estimates:

(1) The result holds for $r=1$.

(2) If $\alpha=1$, the boundedness from $L^{n, 1}$ to $L^{\infty}\left(L^{n}\right)$ holds.

(3) The unmixed estimate $(q=r)$ holds for $1<\alpha<n /(n+1)$ if $\alpha / n \leq$ $1 / r<1-\alpha$ (this is the maximum range allowed in Conjecture 4.1 for $q=r)$.

We observe first that the case $r=1$ corresponds to the usual Riesz potential of order $\alpha$; indeed,

$$
\int_{\mathbf{R}^{n}} \frac{f(x-y)}{|y|^{n-\alpha}} d y=\frac{1}{2} \int_{S^{n-1}} \int_{-\infty}^{\infty} f(x-t u)|t|^{\alpha-1} d t d \sigma(u) .
$$

Then (1) is given by the well-known boundedness of the Riesz potential from $L^{p}$ to $L^{q}$ when $1<p<n / \alpha$ and $n / p-n / q=\alpha$. Observe that if $\alpha=n$, we have the integral of $f$, so that $I_{n}$ is bounded from $L^{1}$ to $L^{\infty}\left(L^{1}\right)$.

(2) has been proved in (3.2). To prove (3), use first the boundedness of the one-dimensional Riesz potential and then the result for the $X$-ray transform given in the preceding section. The precise calculation is as follows:

$$
\begin{aligned}
& \int_{S^{n-1}} \int_{\mathbf{R}^{n}}\left(\int_{-\infty}^{\infty} f(x-t u)|t|^{\alpha-1} d t\right)^{r} d x d \sigma(u) \\
& \quad \leq C \int_{S^{n-1}} \int_{u^{\perp}}\left(\int_{-\infty}^{\infty} f(x-t u)^{\tilde{p}} d t\right)^{r / \tilde{p}} d \lambda_{u^{\perp}}(x) d \sigma(u) \leq C\|f\|_{p}^{r}
\end{aligned}
$$

where

$$
\frac{1}{r}=\frac{1}{\tilde{p}}-\alpha, \quad 1<\frac{r}{\tilde{p}} \leq n+1, \quad \text { and } \quad \frac{n}{p}-\frac{n-1}{r}=\frac{1}{\tilde{p}} .
$$

(The first condition is required for the first inequality, the others for the second one.)

Together with the key estimates we need the following inequalities (for $f \geq 0$ ):

(4) $I_{\alpha} f(x, u) \leq I_{\beta} f(x, u)^{s} I_{\gamma} f(x, u)^{1-s}$ if $\alpha=s \beta+(1-s) \gamma, 0 \leq s \leq 1$, and $0<\beta, \gamma \leq n$

(5) $I_{\alpha} f(x, u) \leq C_{s} M f(x, u)^{s} I_{\gamma} f(x, u)^{1-s}$ if $\alpha=(1-s) \gamma, 0 \leq s<1$. 
The first one is a simple consequence of Hölder's inequality; (5) is a variant of the classical Hedberg's inequality [AH, p. 54] and can be seen as a substitute of (4) for $\beta=0$.

\subsection{The end of the proof.}

To conclude the proof if $\alpha \geq n /(n+1)$, let $r=n / \alpha$ and $p$ in the conjectured range, define $\beta$ in such a way that the line in the plane $(1 / p, 1 / r)$ passing through $(1,1)$ and $(1 / p, \alpha / n)$ cuts the line $p=2 r$ at a point whose second coordinate is $\beta / n$. Use (4) with $\gamma=n$ and (3) to obtain a restricted estimate (that is, for characteristic functions), and interpolate.

If $\alpha<n /(n+1)$ using (1), (2) and (3) plus interpolation we get a range for $p$ and $r$. This range can be improved to cover all the points such that $2 / r \geq 1 / p$. Indeed, we only need to consider some $\gamma>\alpha$ for which the result has been proved for the same couple $(p, r)$, the estimate for $M$ given in Section 2 and (5).

The proof for the case $n=2$ follows from Theorem 2.4 and (5) with $\gamma=n$; the result is obtained for $r<n / \alpha$. Some end-point results (that is, for $r=n / \alpha$ ) can be given using the preceding method.

\section{Radial functions}

The mixed norm inequalities become easier when we restrict to radial functions, even if the range of positive results is larger.

\subsection{Two key estimates.}

The first result of this type we mention is due to A. Carbery, E. Hernández and F. Soria [CHS], and says the following.

Theorem 5.1. The operator $f \mapsto \sup _{u} M f(x, u)$ is bounded from $L^{n, 1}$ to $L^{n}$ and from $L^{p}$ to $L^{p}$ if $p>n$, when restricted to radial functions.

An alternative proof appears in [DNO], where we proved the following pointwise inequality:

$$
M \chi_{E}(x, u) \leq C(n)\left(\chi_{E}\right)^{*}(x)^{1 / n},
$$

valid when $E$ is a radially symmetric measurable set in $\mathbf{R}^{n}\left(\left(\chi_{E}\right)^{*}\right.$ denotes the $n$-dimensional Hardy-Littlewood maximal operator acting on the characteristic function of $E$ ). From this inequality the case $p=n$ of the theorem follows at once, and $p>n$ is deduced by interpolation.

This theorem gives the case $r=\infty$ of Conjecture 2.1 for $M$ acting on radial functions (remember that the counterexample for general functions is given by the characteristic function of a Besicovitch set). 
To improve the results in Sections 3 and 4 we need another end-point result which is the substitute of Inequality (3.2) and was proved in [DO].

Theorem 5.2. Let $E$ be a measurable, radially symmetric set of finite measure in $\mathbf{R}^{n}$, then

$$
\sup _{u} X \chi_{E}(x, u) \leq C|E|^{1 / n} .
$$

\subsection{Sharp results for radial functions.}

Theorem 5.3. (a) Conjecture 2.1 holds for $M$ and $H$ when $f$ is radial, and for $M$ the case $r=\infty$ is also valid.

(b) The $X$-ray transform satisfies Inequality (1.6) for radial functions if and only if $1 \leq p<n$ and $1 \leq r \leq \infty$.

(c) $I_{\alpha}$ satisfies Inequality (1.10) for radial functions if and only if

$$
1<p<\frac{n}{\alpha}, \quad \frac{n}{p}-\frac{n}{q}=\alpha, \quad \text { and } \quad \frac{n}{p}-\frac{n-1}{r}<1 .
$$

(a) is deduced for $M$ from Theorem 5.1 and interpolation, and Lemma 3.3 can be used to extend the results to $H$.

Theorem 5.2 implies that the $X$-ray transform is bounded from $L^{n, 1}$ restricted to radial functions to $L^{\infty}\left(L^{\infty}\right)$ and (b) is immediate.

Finally, to prove (c) we use (1), (4), and (5) as in the Section 4, and replace (2) by the result mentioned in the preceding paragraph which holds for $I_{1}$.

P. Sjögren and F. Soria proved in $[\mathbf{S j S o}]$ that $M$ restricted to radial functions satisfies a weak-type inequality for $p=1$ and $r=1$. For general functions this result was proved to be false by R. Fefferman [F1]. Together with Theorem 5.1 we deduce that a restricted weak-type result holds on radial functions for $M$ when $p$ and $r$ are on the end-point line $n / p-(n-1) / r=1$.

\section{Other results on directional operators}

\subsection{Finite set of directions.}

Given a subset $E$ of $S^{n-1}$ define the operator

$$
M_{E} f(x)=\sup _{u \in E} M f(x, u) .
$$

When $E$ is a finite set, the boundedness of $M_{E}$ in $L^{p}$ for $1<p \leq$ $\infty$ is immediate, but the interesting question is to determine which is the behaviour of the norm of the operator with respect to the number of directions in $E$. The conjecture is that if $N^{n-1}$ is the cardinality of $E$, then the $L^{p}$ norm of $M_{E}$ satisfies the Inequalities (2.6). This 
result is known only for $n=2$. The proof for an equidistributed set of directions is due to A. Córdoba [Co2] (see also [St]); much later, N. H. Katz $[\mathbf{K 1}]$ proved the general case, for which an alternative proof is due to Alfonseca, Soria and Vargas [ASV].

In higher dimensions only the result for $1<p \leq 2$ seems to be known for the equidistributed case (using the estimate for $p=2$ ) and the general case is open. An answer to this conjecture (even for the equidistributed case) implies the Kakeya maximal function conjecture of Subsection 4.2, which has been proved up to $(n+2) / 2($ when $n \geq 3)$.

\subsection{Some results on infinite sets.}

When $E$ is infinite, the boundedness of $M_{E}$ might hold or not. Only extremal situations are known currently: either $M_{E}$ is bounded on $L^{p}$ for $1<p \leq \infty$ or $M_{E}$ is unbounded for finite $p$. If $E$ is a lacunary set of directions in the plane the first situation holds $[\mathbf{N S W}]$ and the same thing is true if the set of directions is lacunary of finite order $[\mathbf{S j S j}]$; those are essentially the only sets of directions for which boundedness has been proved.

If $E=S^{n-1}$ the operator is unbounded, but one can construct many discrete sets $E$ for which $M_{E}$ is unbounded based on the following observation: if there are $N_{k}$ (almost) equidistributed directions on the set $E$, the norm is bigger than a power of $\log N_{k}$ (the conjecture in the preceding subsection gives a necessary condition); as a consequence, if the set $E$ contains such subsets of directions for a sequence $N_{k}$ tending to infinity the operator $M_{E}$ must be unbounded. For instance, if $E$ is a sequence of directions of slope $\left\{n^{-\alpha}: n=1,2, \ldots\right\}$, then $M_{E}$ is unbounded (there are $C 2^{k / \alpha}$ directions between $2^{-k}$ and $2^{-k+1}$, and the distance between two consecutive directions among them is almost constant).

Let $E$ be a Cantor set of zero measure. It is not lacunary, but it does not satisfy the unboundedness criterion proposed in the preceding paragraph, either. N. H. Katz $[\mathbf{K 2}]$ showed that the operator associated to the ternary Cantor set is unbounded if $p=2$ (hence if $p<2$ ). This negative result has been extended to central Cantor sets of positive Hausdorff measure by K. E. Hare $[\mathbf{H}]$, but the general situation is largely open.

\subsection{Radial functions.}

In the plane the situation is much clearer when we restrict to radial functions. Let $E \subset S^{1}$ and denote by $d(E)$ the upper Minkowski 
dimension of $E$, which is defined as

$$
d(E)=\limsup _{\delta \rightarrow 0^{+}} \frac{\log \mathcal{N}(\delta)}{-\log \delta}
$$

where $\mathcal{N}(\delta)$ is the minimum number of closed intervals of length $\delta$ needed to cover $E$. If $\bar{E}$ has positive Lebesgue measure, $d(E)=1$. If $\bar{E}$ has zero Lebesgue measure, writing $S^{1} \backslash \bar{E}$ as the union of a sequence of disjoint open intervals, $\left\{I_{j}\right\}$, we have an alternative definition of $d(E)$,

$$
d(E)=\inf \left\{\alpha \geq 0: \sum_{j}\left|I_{j}\right|^{\alpha}<\infty\right\} .
$$

The following theorem is proved in $[\mathbf{D V}]$.

Theorem 6.1. When restricted to radial functions $M_{E}$ is bounded on $L^{p}$ if $p>1+d(E)$ and unbounded if $p<1+d(E)$.

If the dichotomy presented above for the general case (either boundedness for all $p>1$ or unboundedness for all finite $p$ ) holds true, all operators associated to sets with $d(E)>0$ will be unbounded. On the other hand, one can construct $E$ such that $d(E)=0$ and $M_{E}$ is unbounded for all finite $p$ on general functions while it is bounded for all $p>1$ on radial functions (take the lacunary sequence of directions of dyadic slope $2^{-j}$ and insert $j$ equidistributed directions between $2^{-j}$ and $2^{-j-1}$, for all $j$ ).

\section{References}

[AH] D. R. Adams ANd L. I. HedBerg, "Function spaces and potential theory", Grundlehren der Mathematischen Wissenschaften 314, Springer-Verlag, Berlin, 1996.

[ASV] M. A. Alfonseca, F. Soria And A. Vargas, A remark on maximal operators along directions in $\mathbf{R}^{2}$, Preprint.

[BS] C. BenNett AND R. Sharpley, "Interpolation of operators", Pure and Applied Mathematics 129, Academic Press, Inc., Boston, MA, 1988.

[C] A. P. Calderón, On the Radon transform and some of its generalizations, in: "Conference on harmonic analysis in honor of Antoni Zygmund", vols. I, II (Chicago, Ill., 1981), Wadsworth Math. Ser., Wadsworth, Belmont, CA, 1983, pp. 673-689.

[CZ1] A. P. CALderón AND A. ZYGmund, On singular integrals, Amer. J. Math. 78 (1956), 289-309.

[CZ2] A. P. CALderón AND A. ZyGmund, On singular integrals with variable kernels, Applicable Anal. 7(3) (1977/78), 221-238. 
[ChS] A. Carbery, E. Hernández and F. Soria, Estimates for the Kakeya maximal operator on radial functions in $\mathbf{R}^{n}$, in: "Harmonic analysis" (Sendai, 1990), ICM-90 Satell. Conf. Proc., Springer, Tokyo, 1991, pp. 41-50.

[Ch] M. Christ, Estimates for the $k$-plane transform, Indiana Univ. Math. J. 33(6) (1984), 891-910.

[CDR] M. Christ, J. Duoandikoetxea and J. L. Rubio de FranCIA, Maximal operators related to the Radon transform and the Calderón-Zygmund method of rotations, Duke Math. J. 53(1) (1986), 189-209.

[Co1] A. Córdoba, The Kakeya maximal function and the spherical summation multipliers, Amer. J. Math. 99(1) (1977), 1-22.

[Co2] A. CóRdobA, The multiplier problem for the polygon, Ann. of Math. (2) 105(3) (1977), 581-588.

[CM] M. Cowling AND G. MAUCERI, Inequalities for some maximal functions. I, Trans. Amer. Math. Soc. 287(2) (1985), 431-455.

[Dr1] S. W. DruRY, $L^{p}$ estimates for the X-ray transform, Illinois J. Math. 27(1) (1983), 125-129.

[Dr2] S. W. DruRY, A survey of $k$-plane transform estimates, in: "Commutative harmonic analysis" (Canton, NY, 1987), Contemp. Math. 91, Amer. Math. Soc., Providence, RI, 1989, pp. $43-55$.

[D] J. Duonndikoetxea, "Fourier Analysis", Graduate Studies in Mathematics 29, American Mathematical Society, Providence, RI, 2001.

[DNO] J. Duoandikoetxea, V. Naibo and O. Oruetxebarria, $k$-plane transforms and related operators on radial functions, Michigan Math. J. 49(2) (2001), 265-276.

[DO] J. Duoandikoetxea and O. Oruetxebarria, Mixed norm inequalities for directional operators associated to potentials, $P_{o}$ tential Anal. 15(3) (2001), 273-283.

[DV] J. Duoandikoetxea And A. Vargas, Directional operators and radial functions on the plane, Ark. Mat. 33(2) (1995), 281-291.

[F1] R. Fefferman, A theory of entropy in Fourier analysis, Adv. in Math. 30(3) (1978), 171-201.

[F2] R. FefFerman, On an operator arising in the CalderónZygmund method of rotations and the Bramble-Hilbert lemma, Proc. Nat. Acad. Sci. U.S.A. 80(12) (1983), 3877-3878.

[H] K. E. Hare, Maximal operators and Cantor sets, Canad. Math. Bull. 43(3) (2000), 330-342. 
[K1] N. H. Katz, Maximal operators over arbitrary sets of directions, Duke Math. J. 97(1) (1999), 67-79.

[K2] N. H. KATZ, A counterexample for maximal operators over a Cantor set of directions, Math. Res. Lett. 3(4) (1996), 527-536.

[NSW] A. Nagel, E. M. Stein And S. Wainger, Differentiation in lacunary directions, Proc. Nat. Acad. Sci. U.S.A. 75(3) (1978), 1060-1062.

[OS] D. M. Oberlin and E. M. Stein, Mapping properties of the Radon transform, Indiana Univ. Math. J. 31(5) (1982), 641-650.

[SjSj] P. SJöGren And P. SJölin, Littlewood-Paley decompositions and Fourier multipliers with singularities on certain sets, Ann. Inst. Fourier (Grenoble) 31(1) (1981), 157-175.

[SjSo] P. SJÖGren and F. SorIA, Rough maximal functions and rough singular integral operators applied to integrable radial functions, Rev. Mat. Iberoamericana 13(1) (1997), 1-18.

[S1] E. M. SteIn, "Singular integrals and differentiability properties of functions", Princeton Mathematical Series 30, Princeton University Press, Princeton, NJ, 1970.

[S2] E. M. SteIN, "Harmonic analysis: real-variable methods, orthogonality, and oscillatory integrals", Princeton Mathematical Series 43, Monographs in Harmonic Analysis III, Princeton University Press, Princeton, NJ, 1993.

[St] J-O. StrömberG, Maximal functions associated to rectangles with uniformly distributed directions, Ann. of Math. (2) 107(2) (1978), 399-402.

[W1] T. WolfF, An improved bound for Kakeya type maximal functions, Rev. Mat. Iberoamericana 11(3) (1995), 651-674.

[W2] T. WolfF, Recent work connected with the Kakeya problem, in: "Prospects in mathematics" (Princeton, NJ, 1996), Amer. Math. Soc., Providence, RI, 1999, pp. 129-162.

[W3] T. WolfF, A mixed norm estimate for the X-ray transform, Rev. Mat. Iberoamericana 14(3) (1998), 561-600.

Departamento de Matemáticas

Universidad del País Vasco

Apartado 644

48080 Bilbao

Spain

E-mail address: mtpduzuj@lg.ehu.es 